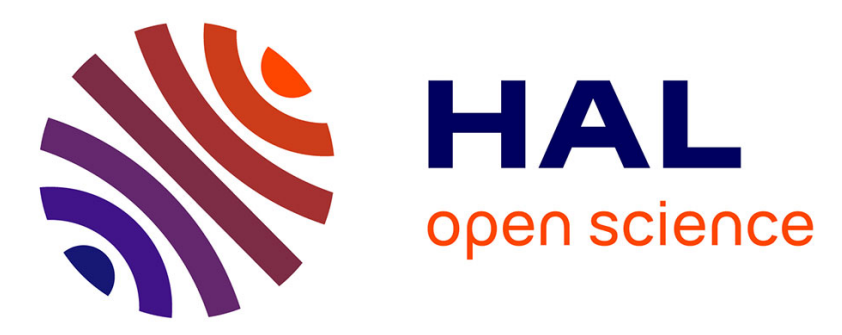

\title{
Nanoforce estimation with Kalman filtering applied to a force sensor based on diamagnetic levitation.
}

\author{
Emmanuel Piat, Joël Abadie, Stéphane Oster
}

\section{To cite this version:}

Emmanuel Piat, Joël Abadie, Stéphane Oster. Nanoforce estimation with Kalman filtering applied to a force sensor based on diamagnetic levitation.. IEEE International Conference Intelligent Robots and Systems, IROS'11., Sep 2011, San Francisco, United States. pp.39-44. hal-00691566

\section{HAL Id: hal-00691566 \\ https://hal.science/hal-00691566}

Submitted on 10 May 2012

HAL is a multi-disciplinary open access archive for the deposit and dissemination of scientific research documents, whether they are published or not. The documents may come from teaching and research institutions in France or abroad, or from public or private research centers.
L'archive ouverte pluridisciplinaire HAL, est destinée au dépôt et à la diffusion de documents scientifiques de niveau recherche, publiés ou non, émanant des établissements d'enseignement et de recherche français ou étrangers, des laboratoires publics ou privés. 


\title{
Nanoforce estimation with Kalman filtering applied to a force sensor based on diamagnetic levitation
}

\author{
Emmanuel Piat and Joël Abadie and Stéphane Oster
}

\begin{abstract}
Nano force sensors based on passive diamagnetic levitation with a macroscopic seismic mass are a possible alternative to classical Atomic Force Microscopes when the force bandwidth to be measured is limited to a few Hertz. When an external unknown force is applied to the levitating seismic mass, this one acts as a transducer that converts this unknown input into a displacement that is the measured output signal. Because the little damped and long transient response of this kind of macroscopic transducer can not be neglected, it is then necessary to deconvolve the output to correctly estimate the unknown input force. The deconvolution approach proposed in this article is based on a Kalman filter that use an uncertain a priori model to represent the unknown nanoforce to be estimated. The main advantage of this approach is that the end-user can directly control the unavoidable trade-off that exists between the wished resolution on the estimatedforce and the response time of the estimation.
\end{abstract}

\section{INTRODUCTION}

The design of micro and nano force sensors is constrained by the fact that only force effects can be directly observed. Because of this, a transducer is necessary to convert the force into a measurable effect. The force is the unknown input to reconstruct and the effect is the measured output signal. Most of the time, the measured force effect is related to a displacement $x$ and the usual scalar expression used to calculate the component $F$ of the applied force $\vec{F}$ in one direction $\vec{x}$ of space simply consists in the equation:

$$
F=K x \quad K>0
$$

in which $K$ is the mechanical stiffness of the transducer along $\vec{x}$ (by convention $x$ is set to zero when there is no displacement). This steady-state equation supposes that the transient response of the transducer can be neglected. This is usually considered to be the case for classical designs using monolithic elastic microstructures like microcantilevers [1]: AFM based microforce sensors [2] [3], piezoresistive microforce sensors [4], capacitive microforce sensors [5], piezoelectric microforce sensors [6], etc. When the transient dynamic of the transducer due to the evolution of the successive derivatives of $x$ is not negligeable, (1) can not be used and the general framework of the force reconstruction corresponds in fact to a deconvolution problematic of a noisy output signal. In the specific case treated in this article, the unknown input is a nanoforce that is applied to a macroscopic seismic mass that levitates passively thanks to the diamagnetic levitation principle. This seismic mass acts

FEMTO-ST Institute

CNRS - UFC - ENSMM - UTBM

Besançon - France

epiat@ens 2 m.fr as a transducer that converts the unknown input force into a displacement that is the measured output signal corrupted by noise. This kind of macroscopic transducer has a badly damped and long transient response, thus this dynamic behaviour must be taken into account during the estimation process contrary to (1). The estimation computation is based on a discrete Kalman filter that use an uncertain a priori model to represent the unknown force to be estimated. This article begins by a short description of the force sensor and its dynamic behaviour (state-space modelling). The calibration process is then briefly presented and followed by the development of the unknown input estimation under gaussian assumptions usually used to derive a Kalman filter. To be realistic, some performances of the force estimation obtained are then characterized in a non gaussian framework. Finally, some experimental results are presented.

\section{PASSIVE MICRO AND NANOFORCE SENSOR PROTOTYPE BASED ON DIAMAGNETIC LEVITATION}

\section{A. Sensor description}

Microforce sensors based on "heavy" rigid seismic mass are really uncommon. A force sensor with a range measurement of several millinewtons and based on a mass moving inside a pneumatic linear bearing is described in [7]. The mass is 21.17 grams and the force resolution is 0.5 micronewton. The air friction inside the bearing is assumed small enought to be neglected. Contrary to the last one, the design presented in this article is based on levitation in order to reach the same force resolution than an Atomic Force Microscope (AFM) but with a larger range measurement. This design is based on a lighter macroscopic mass $(\approx 70 \mathrm{mg})$ that is levitating passively thanks to the diamagnetic levitation principle. This mass is a rigid ten centimeters long capillary tube made of glass on which are stuck two small magnets $M_{2}$. The whole structure is called maglevtube (Figure 1). As it is shown in figure 2 , the maglevtube levitates passively around a given equilibrium state thanks to repulsive diamagnetic effects (generated by the graphite diamagnetic plates) coupled with attractive magnetic effects (generated by the magnets $M_{1}$ and $\left.M_{1}^{\prime}\right)$. The maglevtube has a microscopic tip on which is applied the unknown external force $\vec{F}$. The sensor is currently designed to only measure forces applied along the longitudinal axis $\vec{x}$ of the tube. Thus, the unknown force $\vec{F}$ is assumed to be colinear to $\vec{x}$ and has the following components in the global reference frame $R_{0}$ given in figure 2:

$$
\vec{F}\left[\begin{array}{lll}
F^{x} & 0 & 0
\end{array}\right]^{\top}
$$




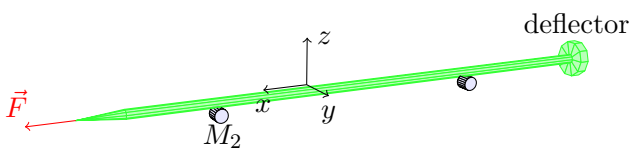

Fig. 1. Macroscopic seismic mass sensitive to external forces.

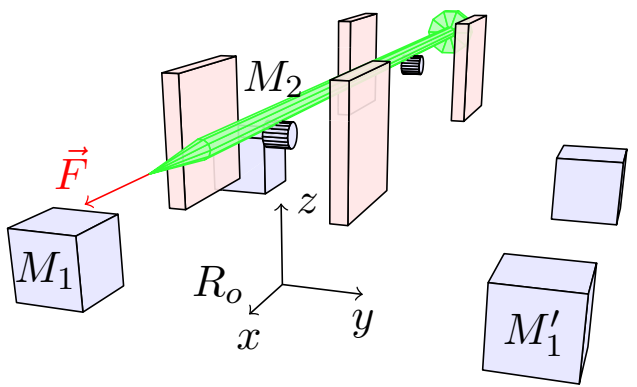

Fig. 2. Levitating seismic mass in the force sensing device that is using magnets $M_{1}, M_{1}^{\prime}, M_{2}$ and diamagnetic graphite plates.

\section{B. Force sensing principle}

When the force $F^{x}$ is applied to the tube tip, the displacement obtained corresponds to a little damped behaviour because the viscous friction due to the air is very small. The simulated displacement computed with Matlab-Simulink and obtained with a force $F^{x}$ set to one micronewton is given in figure 3 (step response). This simulation of the prototype presented in section II-C is done with a complete computation of the internal magnetic and diamagnetic forces at each time step of the Simulink solver. Thus, the complete behaviour of the six dof of the maglevtube can be plotted if necessary. The settling time at 5\% along $\vec{x}$ axis is typically 20 secondes. Overshoot is $97 \%$. The nonlinear steady-state response of the maglevtube is given in figure 4 ("force versus displacement" characteristic). The slope of this curve corresponds to the magnetic stiffness $K_{m}^{x}$ of the sensor that is equivalent to an invisible magnetic spring with a small damping. One can notice that the linearity of the stiffness is good with displacements between \pm 1.5 millimeters. For such range of displacements, the maximum relative error between the linearized force and the nonlinear magnetic force is $0.63 \%$ in this simulation. Knowing the magnetic stiffness $K_{m}^{x}$, the force measurement is given by (1) in steady-state:

$$
F^{x}=K_{m}^{x} x \quad K_{m}^{x}>0 .
$$

with $x$ the displacement of the maglevtube measured with an external distance sensor. Because the stiffness is equal to $0.0289 \mathrm{~N} / \mathrm{m}$ in this simulation, the corresponding measured force range associated to a \pm 1.5 millimeters range displacement is $\pm 43 \mu \mathrm{N}$. A more complete description of this sensor can be found in [8].

\section{Experimental prototype}

Typical $K_{m}^{x}$ stiffnesses obtained with the prototype shown on figure 5 are between $0.005 \mathrm{~N} / \mathrm{m}$ and $0.03 \mathrm{~N} / \mathrm{m}$ (same order of magnitude than for very flexible AFM cantilevers). The stiffness can be easily adjusted by changing the distance

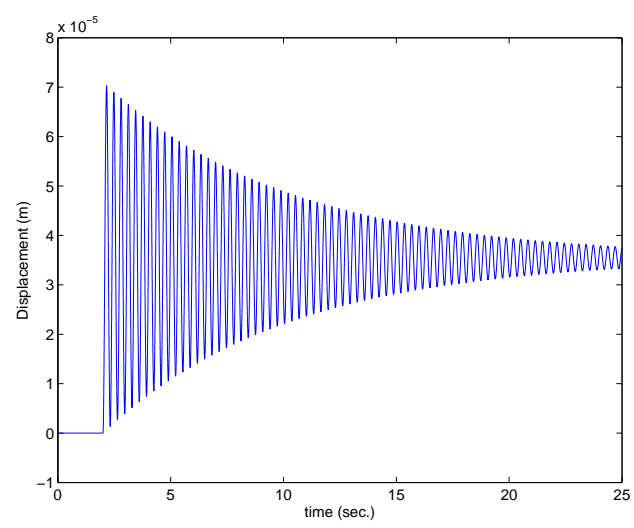

Fig. 3. Simulated step response of the maglevtube with an external force set to one micronewton.

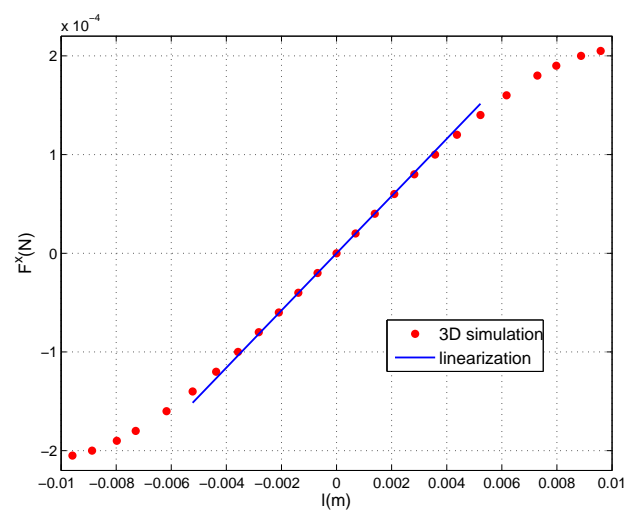

Fig. 4. Force versus displacement steady-state characteristic.

between magnets $M_{1}$ and $M_{1}^{\prime}$. Lower is the stiffness and better is the sensitivity of the sensor. There is nevertheless a limitation on the lower value that can be reached for $K_{m}^{x}$ because if magnets $M_{1}$ and $M_{1}^{\prime}$ are too far away from each other, the magnetic force along the vertical axis is not sufficient to compensate the maglevtube weight. Typical mass $m$ for the maglevtube is around $70 \mathrm{mg}$. Typical resonant frequency is around $3 \mathrm{~Hz}$. The sensor used to measure the maglevtube displacement is a confocal chromatic sensor (manufactured by STIL SA) that is aimed at a glass deflector stuck at the rear of the maglevtube (see figure 1). Typical standard deviation for a CL2 confocal head is $12 \mathrm{~nm}$. Thus, without any signal processing and in steady state, the minimal standard deviation that can be expected for the force is $0.12 \mathrm{nN}$ if $K_{m}^{x}=0.01 \mathrm{~N} / \mathrm{m}$. In practice, such small values can not be reached because of the seismic mass sensitivity to seismic disturbances (subsonic air disturbances are avoided by enclosing the sensor with a chamber). Stochastic low frequency seismic vibrations of magnets $M_{1}$ and $M_{1}^{\prime}$ generate unwanted magnetic return forces that are applied on magnets $M_{2}$ and a stochastic oscillating behaviour of the maglevtube results. With a massive concrete ground slab to minimise seismic vibrations, the minimal standard deviation currently reached is $30 \mathrm{~nm}$ (measured with a CL2 head). 


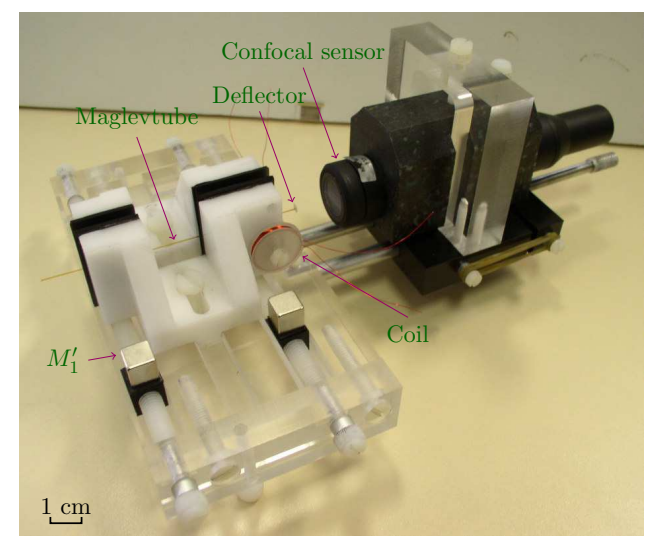

Fig. 5. Force sensor prototype.

\section{SENSOR MODELLING AND CALIBRATION}

\section{A. One dof linear modelling of the maglevtube dynamic}

Let $G$ the centre of gravity of the maglevtube and $x$ its position in the frame $R_{0}$ (cf. figure 2). Coordinate $x$ is set to zero when the maglevtube is in steady state without any external excitation. If an external force $F^{x}$ is applied under assumption (2) to the maglevtube tip, the dynamic of $G$ along $\vec{x}$ can be modelled by:

$$
m \ddot{x}=F^{x}+F_{m a g}^{x}+F_{v i s c}^{x}
$$

in which $m$ is the maglevtube mass and $F_{v i s c}^{x}$ is the visquous friction force due to the air. If the displacement of the tube along axis $\vec{x}$ remains in the linear domain given in section II-B and if the speed is small, equation (4) becomes:

$$
m \ddot{x}=F^{x}-K_{m}^{x} x-K_{v}^{x} \dot{x}
$$

where $K_{m}^{x}$ is the magnetic stiffness and $K_{v}^{x}$ the viscous damping coefficient. A possible state equation associated to (5) with $X(t)=\left[\begin{array}{ll}x & \dot{x}\end{array}\right]^{\top}$ and $x(t)$ as output is then:

$$
\begin{gathered}
\dot{X}(t)=A X(t)+B F^{x}(t) \\
x(t)=C X(t) \\
A=\left[\begin{array}{cc}
0 & 1 \\
-\frac{K_{m}^{x}}{m} & -\frac{K_{v}^{x}}{m}
\end{array}\right] \quad B=\left[\begin{array}{c}
0 \\
\frac{1}{m}
\end{array}\right] \quad C=\left[\begin{array}{ll}
1 & 0
\end{array}\right]
\end{gathered}
$$

\section{B. Calibration}

Calibration is usually a complex problem for micro and nano force sensors based on elastic microstructures because of the lack of standard forces at this scale. Stiffness absolute uncertainty is most of the time not specified and is still an open problem on which are working international metrology laboratories [9]. Calibrating micro force sensors based on macroscopic seismic mass is easier and several dynamic calibration methods have been investigated. They are based on particular external force generation like impact force [10], step force [11] and oscillating force [12], [13]. Because the maglevtube mass $m$ can be easily measured with a precision balance, a Zero Input Response (ZIR) is another possible way to identify the two others parameters $K_{m}^{x}$ and $K_{v}^{x}$. It

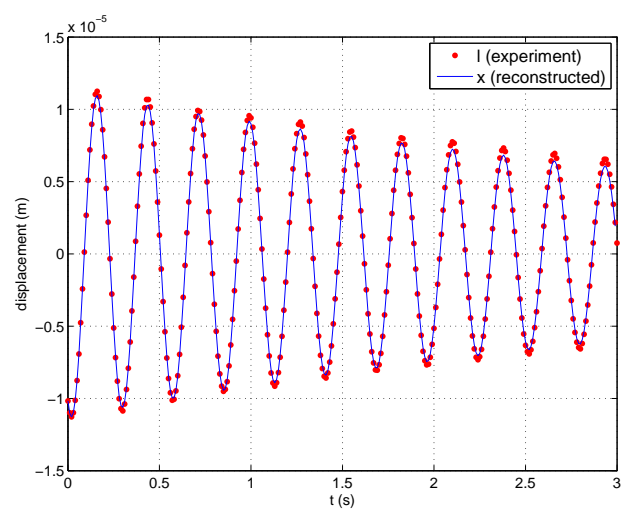

Fig. 6. Measured and reconstructed zero input response (ZIR) of the maglevtube displacement.

requires an unknown excitating force $F^{x}$ with the following dynamic

$$
\begin{cases}F^{x}(t) \neq 0 & t_{0} \leq t<t_{1} \quad \forall F^{x} \\ F^{x}(t)=0 & t \geq t_{1}\end{cases}
$$

which can be generated using two coils located near the rear diamagnetic plates (see figure 5). Knowing $m$, figure 6 shows the matching between both experimental ZIR and the linear model after the parametric identification of $K_{m}^{x}$ and $K_{v}^{x}$ in (5) with $F^{x}$ set to zero (done with Matlab identification toolbox which also estimate initial conditions).

\section{UNKNOWN INPUT FORCE ESTIMATION}

Despite the fact that the model-based deconvolution framework of a noisy output is an ill-posed problem with no exact solution, numerous approaches have been developped in the past such as for instance the Wiener deconvolution filter or deconvolution methods based on regularization [14]. In the specific context of micro or nanoforce measurement in microrobotics, the deconvolution problematic has been little addressed but some alternative approaches using unknown input observers have been recently published [15]. These approaches generally requires to set several parameters. The method proposed here only requires one parameter to adjust an intuitive trade-off (like in regularization methods) between the wished resolution of the force estimated and the response time of the estimation.

\section{A. A priory force modelling}

The noisy measurement $y_{k}^{m}$ of the maglevtube displacement with the confocal chromatic sensor is done with a sampling rate $T_{s}$ at each sampling time $t_{k}=k T_{s}$. The estimation of the unknown force with the set $\left\{y_{k}^{m}\right\}_{k \geq 1}$ is done at each sampling time $t_{k}$. It is based on an a priori discrete-time stochastic model for the force evolution that will be processed inside a recursive discrete Kalman filter. The working out of this a priori model is based on the discretization of a Wiener process:

$$
\dot{F}(t)=\omega(t)
$$


$F(t)$ is a model for the real force $F^{x}(t)$ and $\omega(t)$ is a stationary zero-mean infinite-variance white gaussian stochastic process representing the fact that the evolution of the force derivative is not known. The autocorrelation function $\phi_{\omega, \omega}$ of this stationary process is characterized by its power spectral density $W_{\dot{F}}$ :

$$
\phi_{\omega, \omega}(\tau)=W_{\dot{F}} \delta(\tau) \quad \forall \tau \in \mathbb{R}
$$

The term $W_{\dot{F}}$ is a parameter to set by the end-user that will influence in a given way the dynamic of the unknown force estimation (see section V). To estimate with a discrete Kalman filter the external force $F^{x}$ at each sampling time $t_{k}$, a discrete model of the continuous dynamic (6)-(7) is necessary. This discretized model also includes the discretization of the modeled force $F(t)$ thus a concatenation of the process generating the force and the maglevtube dynamic must be considered. This extended system is represented by the following extended stochastic state:

$$
X^{e}(t)=\left[\begin{array}{lll}
x & \dot{x} & F
\end{array}\right]^{\top}
$$

The associated state-space model is obtained with (10) and (6) in which the unknown input force $F^{x}(t)$ is replaced by the modelled random variable $F(t)$ :

$$
\begin{aligned}
\dot{X}^{e}(t) & =\mathcal{A} X^{e}(t)+\mathcal{M} \omega(t) \\
x(t) & =\mathcal{C} X^{e}(t)
\end{aligned}
$$

with

$$
\mathcal{A}=\left[\begin{array}{ccc}
A_{11} & A_{12} & B_{11} \\
A_{21} & A_{22} & B_{21} \\
0 & 0 & 0
\end{array}\right] \quad \mathcal{M}=\left[\begin{array}{l}
0 \\
0 \\
1
\end{array}\right] \mathcal{C}=\left[\begin{array}{lll}
1 & 0 & 0
\end{array}\right]
$$

The state equation (13) is driven by $\omega(t)$ and thus by the parameter $W_{\dot{F}}$ to set. Its discretization using a zero-order hold (zoh) on $\omega(t)$ gives:

$$
\begin{aligned}
X_{k+1}^{e} & =\mathcal{F} X_{k}^{e}+\Omega_{k} \\
x_{k} & =\mathcal{C} X_{k}^{e}
\end{aligned}
$$

with

$$
X_{k}^{e}=\left[\begin{array}{lll}
x_{k} & \dot{x}_{k} & F_{k}
\end{array}\right]^{\top} \quad \Omega_{k}=\left[\begin{array}{lll}
\omega_{k}^{x} & \omega_{k}^{\dot{x}} & \omega_{k}^{F}
\end{array}\right]^{\top}
$$

and

$$
\mathcal{F}=e^{\mathcal{A} T_{s}}=\left[\begin{array}{ccc}
\mathcal{F}_{11} & \mathcal{F}_{12} & \mathcal{F}_{13} \\
\mathcal{F}_{21} & \mathcal{F}_{22} & \mathcal{F}_{23} \\
0 & 0 & 1
\end{array}\right]
$$

$\Omega_{k}$ is a discrete-time band-limited white gaussian random process with zero-mean characterizing uncertainties on $x_{k}$, $\dot{x}_{k}$ and $F_{k}$ due to the stochastic force model used and the zoh. Its $3 \times 3$ covariance matrix $Q$ is:

$$
\begin{aligned}
Q=E\left[\Omega_{k} \Omega_{k}^{\top}\right] & =\int_{0}^{T_{s}} e^{\mathcal{A} t} \mathcal{M} W_{\dot{F}} \mathcal{M}^{\top} e^{\mathcal{A}^{\top} t} d t \\
& =W_{\dot{F}} \int_{0}^{T_{s}} e^{\mathcal{A} t} \mathcal{M} \mathcal{M}^{\top} e^{\mathcal{A}^{\top} t} d t \\
& =W_{\dot{F}} \eta\left(T_{s}\right)
\end{aligned}
$$

In (22), $Q$ is proportional to $W_{\dot{F}}$ and it can be easily shown with (21) that the variance of $\omega_{k}^{F}$ is equal to

$$
\sigma^{2}\left(\omega_{k}^{F}\right)=Q_{33}=T_{s} W_{\dot{F}}
$$

The evolution of $F_{k}$ (third component of $X_{k}^{e}$ ) is obtained from (16) and (18):

$$
F_{k+1}=F_{k}+\omega_{k}^{F} \quad k \geq 0
$$

and the statistic properties of the random process $\omega_{k}^{F}$ are

$$
\begin{gathered}
E\left[\omega_{k}^{F}\right]=0 \quad E\left[\left(\omega_{k}^{F}\right)^{2}\right]=\sigma^{2}\left(\omega_{k}^{F}\right)=T_{s} W_{\dot{F}} \\
E\left[\omega_{i}^{F} \omega_{j}^{F}\right]=\sigma^{2}\left(\omega_{k}^{F}\right) \delta_{i j}=T_{s} W_{\dot{F}} \delta_{i j}
\end{gathered}
$$

Equations (24) to (26) fully characterize the a priori discretetime gaussian stochastic model that will be used inside the Kalman filter. The uncertainty model (24) corresponds to a discrete-time gaussian random walk that is usually written as follow (index shift on $\omega_{k}^{F}$ ):

$$
F_{k}=F_{k-1}+\omega_{k}^{F} \quad k \geq 1
$$

It comes from (27) that

$$
F_{k}=\sum_{i=1}^{k} \omega_{i}^{F}+F_{0}
$$

Because successive random variables $\omega_{i}^{F}$ form an a priori discrete zero-mean white gaussian process (the white property is induced by (26)), $F_{k}$ in (28) is gaussian if the knowledge on $F_{0}$ is assumed gaussian or if $F_{0}$ is supposed equal to some fixed value. Its a priori variance at each step $k$ can be calculated thanks to (26):

$$
\sigma^{2}\left(F_{k}\right)=\sum_{i=1}^{k} \sigma^{2}\left(\omega_{i}^{F}\right)+\sigma^{2}\left(F_{0}\right)=k T_{s} W_{\dot{F}}+\sigma^{2}\left(F_{0}\right)
$$

(29) shows that bigger is the parameter $W_{\dot{F}}$ to set and bigger is the a priori uncertainty (variance) on the possible values of the modelled unknown force $F_{k}$ at time $t_{k}$ and the uncertainty growth in time is linear with $t_{k}$ (see figure 7). So at this early stage, it is possible to say that if the unknown force is supposed to vary rapidly, $W_{\dot{F}}$ that represents the growth rate of the a priori uncertainty on $F_{k}$ should be set greater than if the force is supposed to vary slowly. Finally, the measurement $m_{k}^{x}$ of $x_{k}$ (given by (17)) takes into account the discrete-time white gaussian noise $v_{k}$ with zero-mean and variance $R$ added by the confocal chromatic sensor:

$$
m_{k}^{x}=x_{k}+v_{k}=\mathcal{C} X_{k}^{e}+v_{k}
$$

\section{B. Force estimation using a time-varying Kalman filter}

If the parameter $W_{\dot{F}}$ is changed by the end-user during the force estimation process, a time-varying Kalman filter must be used and a numerical computation of $Q$ must be done each time $W_{\dot{F}}$ is changed (the term $\eta\left(T_{s}\right)$ in (22) can be precomputed [16]). The prediction-estimation stages of the Kalman filter are derived from equations (16) and (30):

$$
\begin{aligned}
& \hat{X}_{k \mid k-1}^{e}=\mathcal{F} \hat{X}_{k-1}^{e} \\
& P_{k \mid k-1}=\mathcal{F} P_{k-1} \mathcal{F}^{\top}+Q \\
& K_{k}=P_{k \mid k-1} \mathcal{C}^{\top}\left(\mathcal{C} P_{k \mid k-1} \mathcal{C}^{\top}+R\right)^{-1} \\
& \hat{X}_{k}^{e}=\hat{X}_{k \mid k-1}^{e}+K_{k}\left(m_{k}^{x}-\mathcal{C} \hat{X}_{k \mid k-1}^{e}\right) \\
& P_{k}=\left(I-K_{k} \mathcal{C}\right) P_{k \mid k-1}
\end{aligned}
$$




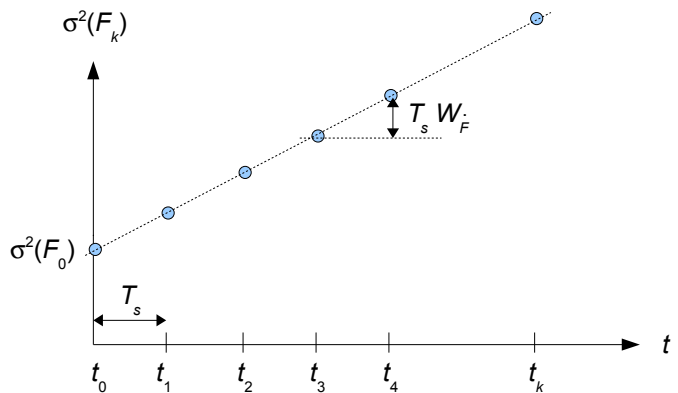

Fig. 7. $W_{\dot{F}}$ effect on the a priori variance $\sigma^{2}\left(F_{k}\right)$

$m_{k}^{x}$ is the noisy measurement of the mavlevtube displacement (input of the Kalman filter). The output of the filter is the estimation $\hat{F}_{k}$ of $F^{x}(t)$ at time $t_{k}$. It is given by:

$$
\hat{F}_{k}=\mathcal{C}_{F} \hat{X}_{k}^{e}
$$

thanks to the output matrix $\mathcal{C}_{F}=\left[\begin{array}{lll}0 & 0 & 1\end{array}\right]$.

The Kalman filter is initialized for instance with the maglevtube in its equilibrium state when no force is applied to it:

$$
\hat{X}_{0}^{e}=\left[\begin{array}{lll}
0 & 0 & 0
\end{array}\right]^{\top}
$$

The covariance matrix $P_{0}$ of the initial estimation error is taken equal to:

$$
P_{0}=\left[\begin{array}{ccc}
\sigma^{2}\left(x_{0}\right) & 0 & 0 \\
0 & \sigma^{2}\left(\dot{x}_{0}\right) & 0 \\
0 & 0 & \sigma^{2}\left(F_{0}\right)
\end{array}\right]
$$

in which each variance represents the a priori uncertainty on $x_{0}, \dot{x}_{0}$ and $F_{0}$. These values are chosen to be coherent with the initial conditions associated to the experiment made. In practice, they have little importance if the user starts the Kalman filter with no force applied on the maglevtube and waits a few seconds such that the Kalman gain $K_{k}$ converges to its steady-state $K_{\infty}\left(W_{\dot{F}}, T_{s}, R\right)$ (solution to the discrete Riccati equation that depends on $W_{\dot{F}}, T_{s}$ and $R$ ) before applying an unknown varying external force.

\section{Simulated Results}

Studying in simulation the estimation behaviour with a force $F^{x}(t)$ really generated by (10) has no interest in practice because this model is purely theorical (it corresponds to a brownian evolution of the input force). The characteristics of $\hat{F}_{k}$ will be illustrated on a canonical input force instead. In this article, we focus only on a step input force. To be independant of (38), a steady-state Kalman filter is used substituting $K_{\infty}$ to $K_{k}$ and using only equations (31) (34) (36). Sampling time $T_{s}$ is $0.001 \mathrm{sec}$. The variance of measurement noise is $R=1.44 \times 10^{-16} \mathrm{~m}^{2}$. The maglevtube parameters are $m=74 \mathrm{mg}, K_{m}^{x}=0.02818 \mathrm{~N} / \mathrm{m}$ (in linear domain), $K_{v}^{x}=1.8 \times 10^{-5} \mathrm{~N} . \mathrm{s} / \mathrm{m}\left(\zeta=6.23 \times 10^{-3}\right)$. Identified values are $K_{m}^{x}=0.02812 \mathrm{~N} / \mathrm{m}, K_{v}^{x}=1.772 \times$ $10^{-5} \mathrm{~N} . \mathrm{s} / \mathrm{m}\left(\zeta=6.14 \times 10^{-3}\right)$. Figures 8 and 9 shows $\hat{F}_{k}$ for $W_{\dot{F}}=10^{-18} \mathrm{~N}^{2} / \mathrm{Hz}$ and $W_{\dot{F}}=10^{-15} \mathrm{~N}^{2} / \mathrm{Hz}$ with $T_{s}$ set to 0.001 seconde. Step amplitude to estimate is $100 \mathrm{nN}$. Smaller is $W_{\dot{F}}$ and smaller is the noise on $\hat{F}_{k}$ but longer

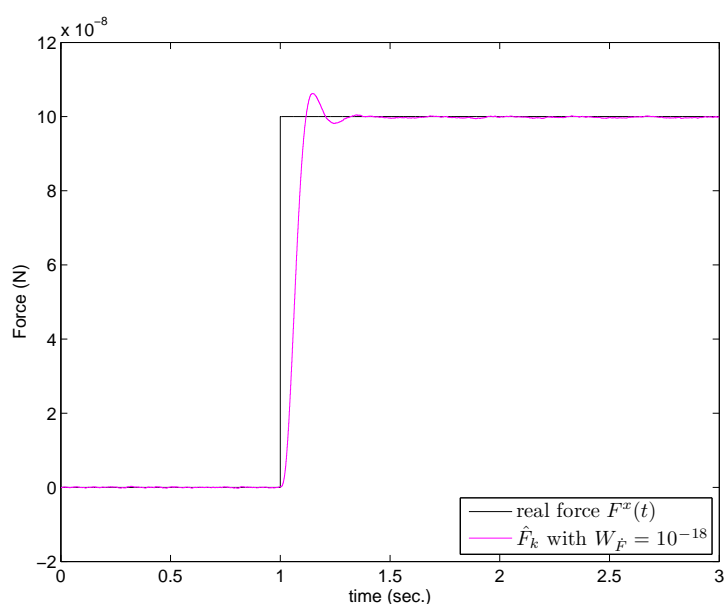

Fig. 8. step-force estimation with $W_{\dot{F}}=10^{-18} \mathrm{~N}^{2} / \mathrm{Hz}$

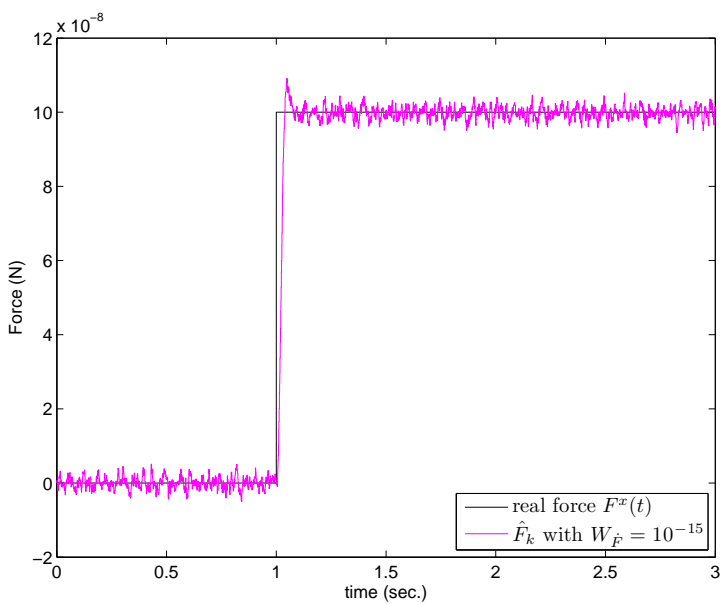

Fig. 9. step-force estimation with $W_{\dot{F}}=10^{-15} \mathrm{~N}^{2} / \mathrm{Hz}$

is the estimation response time. As a consequence, smaller is the amplitude to estimate and smaller must be $W_{\dot{F}}$ to have a good signal to noise ratio in $\hat{F}_{k}$. But in this case, the force bandwidth of the sensor becomes also smaller. This behaviour can be explained with the frequency response $\hat{F}\left(e^{j \omega}\right) / m^{x}\left(e^{j \omega}\right)$ of the steady-state Kalman filter (see figure 10). This frequency response "inverts" the frequency response of the maglevtube with its resonance peak. Bigger is $W_{\dot{F}}$, bigger is the gain in the high frequencies. Thus bigger is the amplification of the high frequency components present in the noise $v_{k}$ inside $m_{k}^{x}$. To reduce this noise level, it is necessary to reduce $W_{\dot{F}}$. But in this case, the high frequency components present in the displacement $m_{k}^{x}$ have a very low amplitude (the maglevtube acts as a low pass filter) and are insufficiently amplified by the kalman filter to correctly reconstruct the high frequency composents in the input $F(t)$. As a consequence, the response time increases (and the bandwith decreases).

\section{EXPERIMENTAL RESULTS}

Figure 11 shows the evolution of the force during a pulloff force measurement. A planar material is pushed against 


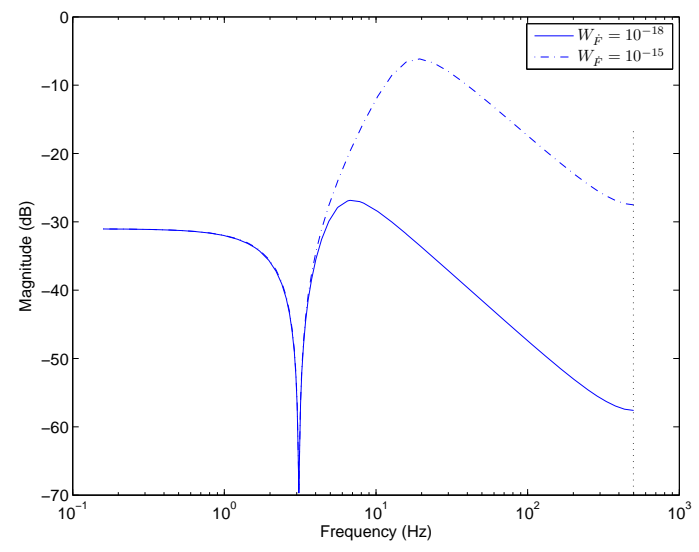

Fig. 10. Impact of $W_{\dot{F}}$ on the tranfer function $\hat{F}\left(e^{j \omega}\right) / m^{x}\left(e^{j \omega}\right)$

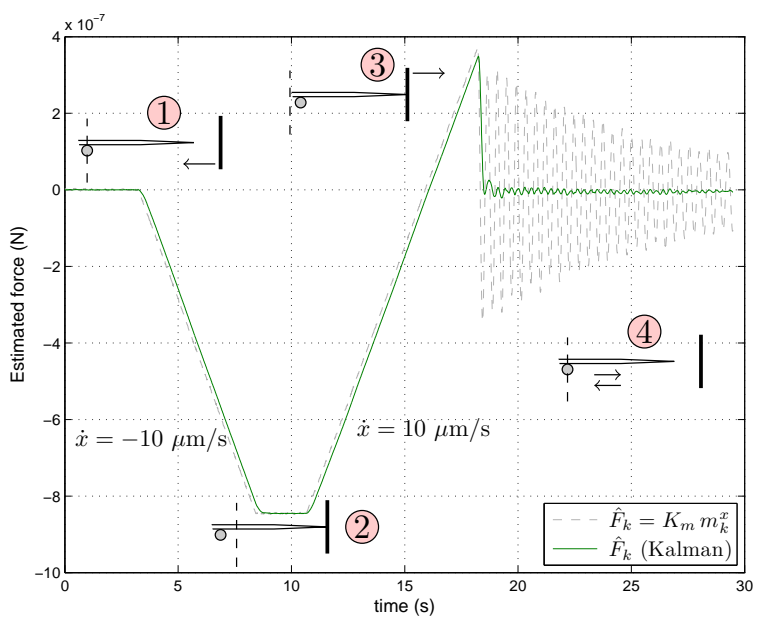

Fig. 11. Experimental pull-off force measurement

a micro-sphere stuck at the maglevtube tip (loading stage) and then pushed back (unloading stage) until the contact is broken between the material and the tip (ZIR displacement that is a one-direction damped oscillating trajectory). After this contact loss, the unknown external force applied on the maglevtube becomes known because it is equal to zero and thus it can be compared with the force estimated with (3) or (36). Equation (3) gives a bad estimation because it is proportional to the ZIR displacement. Kalman estimation (36) gives a better result with a shorter and smaller oscillating transient response. The vertical seismic disturbances of the maglevtube probably participate to these residual oscillations and their modelling is an outlook to this work.

\section{CONCLUSION}

The force estimation presented in this article is based on the displacement of a macroscopic seismic mass. This displacement is processed by a Kalman filter that is using a Wiener process to model the unknown input force. This processing requires the adjustement of a single parameter $W_{\dot{F}}$ which directly adjusts a trade-off between the resolution (variance) of $\hat{F}_{k}$ and the response time of the estimation.
This parameter can be modified at any time by the enduser in accordance with its own knowledge on the force to measure. Compared to simple low-pass filter added on the displacement measurement, the force bandwidth can be extented reasonably four times higher than the displacement bandwidth. This method is computationaly cheap and can be implemented in small DSP or microcontrollers. Response time shorter than 0.1 seconde can be reached with a correct $\mathrm{S} / \mathrm{N}$ ratio despite the very long settling time of the transducer (20 secondes) and its low damping.

\section{ACKNOWLEDGMENT}

This work is supported by the french National Research Agency under STIL $\mu$ FORCE ANR-07-ROBO-0005 contract.

\section{REFERENCES}

[1] M. Sepaniak, P. Datskos, N. Lavrik, and C. Tipple, "Microcantilever transducers: A new approach in sensor technology," Analytical chemistry, pp. 568-575, November 2002.

[2] P. Rougeot, S. Régnier, and N. Chaillet, "Forces analysis for micromanipulation," Proceedings 2005 IEEE international symposium on computational intelligence in robotics and automation, espoo, Finland, pp. 105-110, june 2005 .

[3] N. Kato, I. Suzuki, H. Kikuta, and K. Iwata, "Force-balancing microforce sensor with an optical-fiber interferometer," Review of scientific instruments, vol. 68, pp. 2475-2478, juin 1997.

[4] F. Arai, T. Sugiyama, T. Fukuda, H. Iwata, and K. Itoigawa, "Micro tripaxial force sensor for 3d bio-micromanipulation," In IEEE International Conference on Robotics and Automation (ICRA), 1999.

[5] F. Beyeler, S. Muntwyler, and B. J. Nelson, "A six-axis mems forcethorque sensor with micro-newton and nano-newtonmeter resolution," Journal of Microelectromechanical Systems, vol. 18, pp. 433-441, 2009.

[6] Y. Shen, N. Xi, and W. J. li, "Contact and force control in microassembly," In IEEE 5th International Symposium on Assembly and Task Planning (ISATP), pp. 60-65, 2003.

[7] Y. Fujii, "Method for generating and measuring the micro-newton level forces," Mechanical Systems and Signal Processing, vol. 20, pp. 13621371, 2006.

[8] M. Boukallel, J. Abadie, and E. Piat, "Levitated micro-nano force sensor using diamagnetic levitation," Proc. of the IEEE International Conference of Robotics and Automation, pp. 3219-3224, september 2003.

[9] J. R. Pratt, D. T. Smith, D. B. Newell, J. A. Kramar, and E. Whitenton, "Progress toward système international d'unités traceable force metrology for nanomechanics," J. Mater. Res., vol. 1, pp. 366-379, Jan 2004.

[10] Y. Fujii and H. Fujimoto, "Proposal for an impulse response evaluation method for force transducers," Measurement Science and Technology, vol. 10 (4), pp. N31-N33, 1999.

[11] Y. Fujii, "Proposal for a step response evaluation method for force transducers," Measurement Science and Technology, vol. 14 (10), pp. $1741-1746,2003$

[12] R. Kumme, "Investigation of the comparison method for the dynamic calibration of force transducers," Measurement, vol. 23, pp. 239-245, 1998.

[13] Y. Fujii, "A method for calibrating force transducers against oscillation force," Measurement Science and Technology, vol. 14 (8), pp. 1253 1264, 2003.

[14] G. Demoment, "Image reconstrution and restoration: overview of common estimation structures and problems," IEEE transactions on Acoustics, Speech, and Signal Processing, vol. 37(12), pp. 2024-2036, 1989.

[15] M. Rakotondrabe and P. Lutz, "Force estimation in a piezoelectric cantilever using the inverse-dynamics-based uio technique,', IEEE International Conference on Robotics and Automation (ICRA), pp. 2205-2210, 2009

[16] C. V. Loan, "Computing integrals involving the matrix exponential," IEEE - Trans. Automatic Control, vol. AC-15, October 1970 